\title{
Patients with Increased Levels of the Oxidative Stress Biomarker SOD1 Appear to Have Diminished Postoperative Pain After Midline Laparotomy: A Randomised Trial with Special Reference to Postoperative Pain Score (NRS)
}

\author{
JARI KÄRKKÄINEN ${ }^{1,2}$, TUOMAS SELANDER ${ }^{3}$, MARTIN PURDY ${ }^{1,2}$, \\ PETRI JUVONEN ${ }^{1}$ and MATTI ESKELINEN ${ }^{1,2}$ \\ ${ }^{1}$ Department of Surgery, and ${ }^{3}$ Science Service Center, Kuopio University Hospital, Kuopio, Finland; \\ ${ }^{2}$ School of Medicine, University of Eastern Finland, Kuopio, Finland
}

\begin{abstract}
Background/Aim: The levels of the oxidative stress biomarker superoxide dismutase (SOD1) in plasma in relation to pain at rest 24 hours after $\left(N R S_{24}\right)$ midline laparotomy in patients with rectus sheath block (RSB) analgesia is unknown. Patients and Methods: Initially, 56 patients (39 with cancer), each with an intravenous oxycodone pump as patient-controlled analgesia, were randomized to four groups: control group, no RSB $(n=12)$, single-dose $(n=16)$, repeated-dose $(n=12)$ and continuous infusion ( $n=16)$ of RSB analgesia. The plasma levels of SOD1 were measured directly before, immediately after and 24 hours after surgery. Pain at rest was scored on an 11-point numeric rating scale 24 hours postoperatively (NRS 24: $0=$ no pain to 10=worst pain). Results: The median plasma level of SOD1 increased immediately after operation and this was statistically highly significant $(p=0.007)$. The median plasma level of SOD1 then decreased 24 hours postoperatively and this postoperative decrease was also statistically highly significant $(p<0.001)$. The median plasma levels of SOD1 did not differ significantly between patients with benign disease and those with cancer, preoperatively and after surgery. There was highly significant positive correlation between SOD1 and glutathione peroxidase (GPXI) values postoperatively $(r=0.67, p<0.001)$ and $a$ trend for an inverse correlation between the individual values of the $\mathrm{NRS}_{24}$ and plasma SOD1 values postoperatively in patients with benign disease and those
\end{abstract}

Correspondence to: Matti Eskelinen, MD, Ph.D., School of Medicine, University of Eastern Finland, P.O. Box 100, FI-70029 KYS, Finland. Tel: +358 17173311, Fax: +358 17172611, Mobile: +358 400969444, e-mail: matti.eskelinen@kuh.fi

Key Words: Oxidative stress, SOD1, NRS pain score. with cancer $(r=-0.30, p=0.09)$. Conclusion: Midline laparotomy significantly alters the level of oxidative stress marker SOD1 immediately after surgery, but the level normalizes 24 hours following surgery. Interestingly, patients with increased levels of SOD1 appeared to have diminished postoperative pain after midline laparotomy.

Superoxide dismutase (SOD) enzymes catalyze the reaction where superoxide is dismutated into hydrogen peroxide, and the correct balance between superoxide and hydrogen peroxide is vital for any organism (1). $\mathrm{Cu} / \mathrm{Zn}$ superoxide dismutase (SOD1) is an 32-kDa enzyme that in humans is encoded by the SOD1 gene, located on chromosome 21 . The first identified SOD enzyme was cytoplasmic copper/zinc SOD1; later, mitochondrial iron/manganese SOD (SOD2) and the extracellular SOD SOD3 were discovered (1). SOD1 has an intramolecular disulfide bond and a $\mathrm{Cu} / \mathrm{Zn}$ site in each subunit, which is responsible for dismutation of two $\mathrm{O}^{-}$ anions to $\mathrm{H}_{2} \mathrm{O}_{2}$ and molecular oxygen $(2,3)$.

Yiannakopoulou et al. reviewed the clinical trials that investigated the oxidative stress in open surgery versus laparoscopic surgery (4). They found four trials (5-8) on plasma SOD level and oxidative stress response to surgery and this systematic review aimed to investigate the impact of surgical trauma on oxidative stress. However, there was a great heterogeneity in the measured oxidative markers, methods, time periods and on the types of operation investigated and final conclusions were therefore difficult to draw. The assessment of the SOD1 response, as a biomarker of oxidative stress, to midline laparotomy with rectus sheath block (RSB) analgesia is unknown. Therefore, the aim of this study was to determine the correlation between the plasma level of SOD1 and the pain score at rest 24 hours after midline laparotomy $\left(\mathrm{NRS}_{24}\right)$ under RSB analgesia in patients with benign disease and those with cancer. 


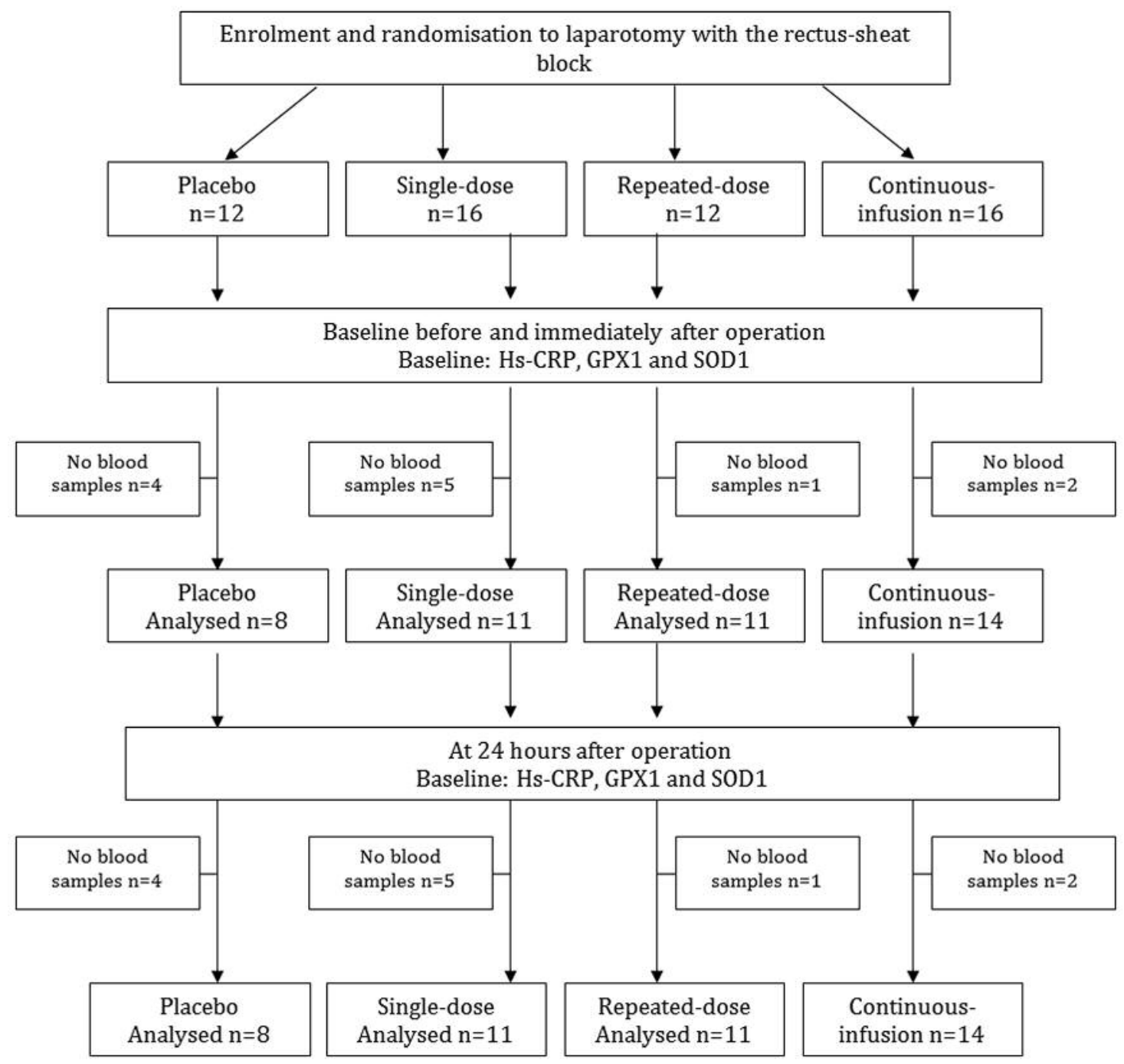

Figure 1. Consort figure of the study design.

\section{Patients and Methods}

The study was approved by the Ethics Committee of Kuopio University Hospital District, Finland (DNRO 120/2011, November 11, 2011), registered in the EudraCT database (EudraCT number 2011005136-25, Consort diagram, Figure 1) and the ClinicalTrials.gov database (ClinicalTrials.gov Identifier: NCT02869841), and was conducted in accordance with the Declaration of Helsinki. Participants gave written consent after receiving verbal and written information on the study. Operations were carried out at Kuopio University Hospital, Kuopio between 2012 and 2015. The CONSORT flowchart of the study is presented in Figure 1. The study design was a prospective, randomized, clinical trial with four parallel groups. Patients undergoing midline laparotomy each had an intravenous oxycodone pump as patient-controlled analgesia, and were then randomized into a control group (no RSB) or into one of three active RSB analgesia groups: single-dose, repeated-dose or continuous infusion of levobupivacaine. The study patients had intravenous oxycodone pumps as patient-controlled analgesia (PCA). The randomization list was generated by computer (www.randomization.com) and a sealedenvelope method was used for blinding and randomization was done preoperatively.

The primary outcome measures were the plasma levels of highsensitivity C-reactive protein (hs-CRP), glutathione peroxidase (GPX1) and SOD1 measured at three time points with highsensitivity assays: before, immediately after, and 24 hours after operation surgery in the control versus those in the three active groups, and the overall pain at rest surveyed and filed on an 11point numeric rating scale at 24 hours following surgery $\left(\mathrm{NRS}_{24}\right.$ : $0=$ no pain to $10=$ worst pain).

All RSB procedures were performed by an experienced surgeon in the operating room before wound closure. The study protocol with exclusion criteria and informed consent were fully described in our earlier original work (9-11). 


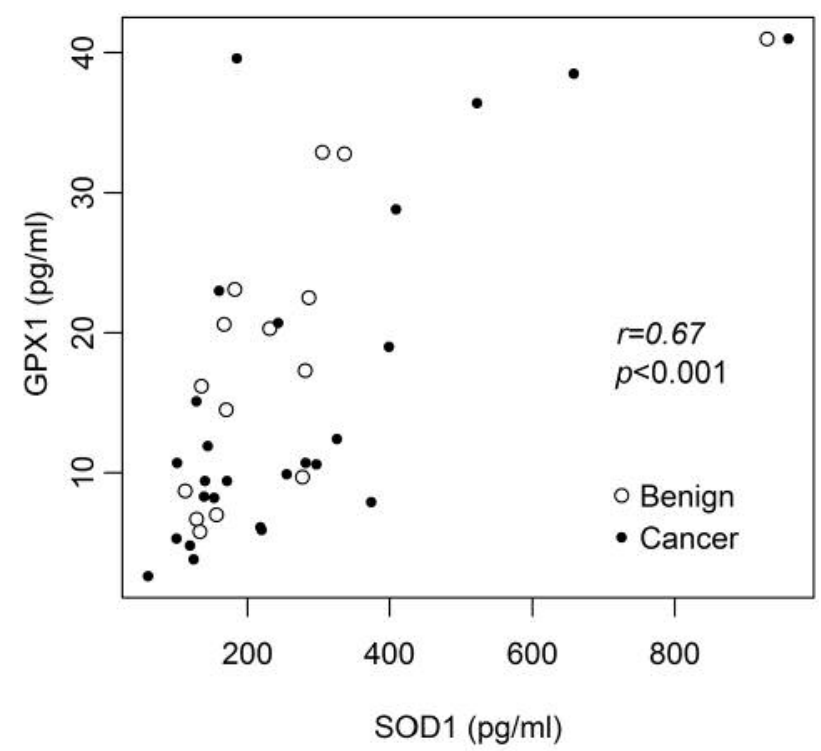

Figure 2. Scatter plots of the postoperative (POP1) plasma values of superoxide dismutase (SOD1) versus glutathione peroxidase (GPX1) in patients with benign disease and those with cancer $(r=0.670, p<0.001)$.

EDTA-blood samples were taken at the prespecified time-points and centrifuged at $1000 \times g$ for $15 \mathrm{~min}$. Plasma was separated and stored frozen at $-70^{\circ} \mathrm{C}$ until analyzed. The plasma GPX1 assays were performed using sandwich-type enzyme-linked immunoassay (ELISA) with BioVendor GPX1 ELISA Kit (Brno, Czech Republic). Plasma hs-CRP was analyzed with a Cobas 6000analyzer (Hitachi, Tokyo, Japan) using the method by Roche Diagnostics (Mannheim, Germany). The plasma SOD1 assays were performed using sandwich-type ELISA with BioVendor $\mathrm{Cu} / \mathrm{Zn}$ SOD ELISA Kit.

The data were entered and analyzed with a statistical software program (IBM SPSS Statistics 22.0; IBM Corp., Armonk, NY, USA). Baseline characteristics between groups were tested by Fisher exact test and then analysis of continuous variables was performed by analysis of variance (ANOVA). Group differences at the three time points were tested by Kruskall-Wallis test and MannWhitney $U$-test or Wilcoxon signed-rank test. The results of the laboratory measurements are presented as the median with interquartile range as distributions were right skewed. A two-sided $p$-value of less than 0.05 was considered statistically significant. The results of the individual GPX1 and SOD1 values immediately postoperatively for patients are shown as scatter plots with Spearman's correlation coefficients in Figure 2. The results of the individual SOD1 values 24 hours postoperatively versus the postoperative $\mathrm{NRS}_{24}$ values for patients are shown as scatterplots with Spearman's correlation coefficients in Figure 3.

\section{Results}

The patients' clinical data were similar in the four study groups, but body mass index and body weight were significantly higher in patients in the single dose and control

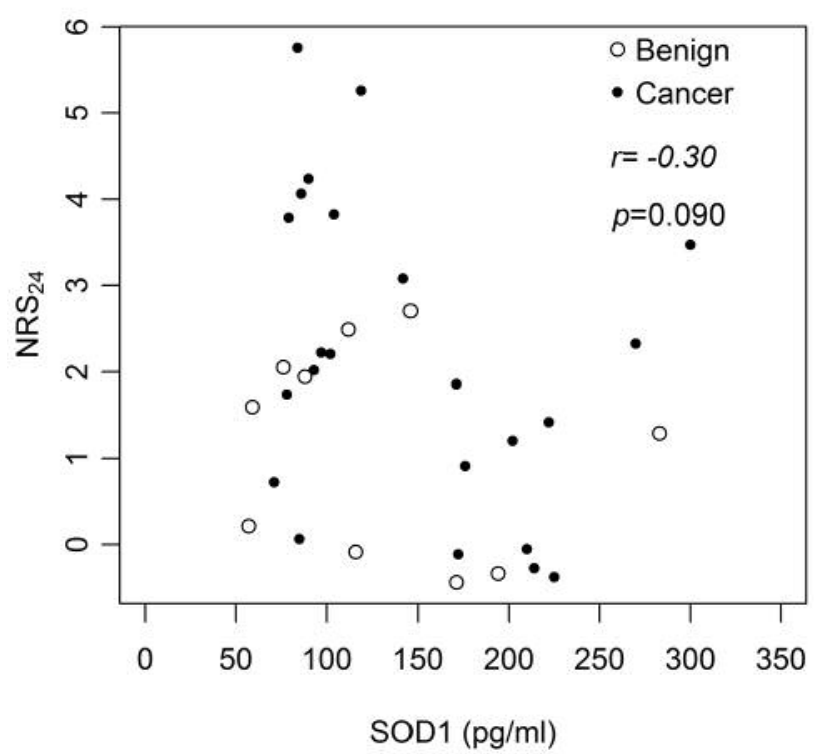

Figure 3. Scatter plots of the individual postoperative numeric rating scale at rest 24 hours postoperatively $\left(\mathrm{NRS}_{24}\right)$ values versus postoperative (POP2) plasma values of superoxide dismutase (SOD1) in patients with benign disease and those with cancer $(r=-0.30, p=0.09)$.

group than in the other two study groups (Table I). The study group consisted of 15 patients with benign disease, nine patients with gastrointestinal cancer, 17 patients with gynecological cancer and three patients with other malignancy (Table I).

The median plasma levels of SOD1 increased by $\sim 13 \%$ immediately after surgery and this increase was statistically highly significant ( $p=0.007$, Table II). The median SOD1 level then decreased by $\sim 45 \%$ at 24 hours postoperatively $(p<0.001$, Table II). However, no differences were detected in the SOD1 values between the control and the three active groups preoperatively and after operation. The patients in the singledose group had a trend for a lower median SOD1 value 24 hours after surgery compared to each of the other three groups (Table III). The median plasma SOD1 level preoperatively or after operation did not differ significantly between patients with cancer and those with benign disease however (Table IV). There was significant correlation between the SOD1 and GPX1 values immediately after operation and 24 hours postoperatively ( $\mathrm{r}=0.67$ and $\mathrm{r}=0.63$, respectively, Figure 2, $p<0.001)$ and a trend for inverse correlation between the individual values of the $\mathrm{NRS}_{24}$ and plasma SOD1 values 24 hours postoperatively in patients with cancer and those with benign disease $(\mathrm{r}=-0.30, p=0.09$, Figure 3 ). The median plasma level of hs-CRP increased only slightly immediately after surgery and this increase was not statistically significant $(p=0.274$, Table II). The median hs-CRP level then increased by 57 fold at 24 hours postoperatively $(p<0.001$, Table II). 
Table I. Clinical data in the four study groups of patients undergoing midline laparotomy. Data are mean (standard deviation) or number of cases*.

\begin{tabular}{|c|c|c|c|c|c|}
\hline \multirow[b]{2}{*}{ Variable } & \multicolumn{5}{|c|}{ RSB method } \\
\hline & Control $n=8$ & Single $n=11$ & Repeated $n=11$ & Continuous $n=14$ & $p$-Value \\
\hline Age (years) & $62.6(14.3)$ & $60.8(12.6)$ & $63.3(10.8)$ & $58.0(10.1)$ & 0.74 \\
\hline Sex: male/female* & $4 / 4$ & $4 / 7$ & $2 / 9$ & $2 / 12$ & 0.22 \\
\hline Height $(\mathrm{cm})$ & $166.6(8.6)$ & $168.4(7.9)$ & $165.7(7.2)$ & $164.3(6.6)$ & 0.62 \\
\hline Weight (kg) & $78.6(11.8)$ & $83.7(12.8)$ & $67.8(13.7)$ & $68.8(10.6)$ & 0.007 \\
\hline BMI $(\mathrm{kg} / \mathrm{m} 2)$ & $28.3(3.8)$ & $29.6(4.4)$ & $24.6(4.3)$ & $25.7(4.9)$ & 0.03 \\
\hline Time in the operative room ( $\mathrm{min}$ ) & $229.4(113.4)$ & $274.9(148.4)$ & $235.7(112.0)$ & $279.7(178.5)$ & 0.85 \\
\hline Operative time $(\mathrm{min})$ & $209.6(141.2)$ & $221.8(156.4)$ & $154.4(95.0)$ & $253.3(168.9)$ & 0.55 \\
\hline Perioperative bleed (ml) & $696(741)$ & $822(906)$ & $697(967)$ & $1340(928)$ & 0.31 \\
\hline ASA $1 / 2 / 3 / 4 *$ & $0 / 6 / 2 / 0$ & $0 / 7 / 3 / 1$ & $0 / 5 / 6 / 0$ & $2 / 7 / 5 / 0$ & 0.43 \\
\hline Length of skin incision(s) (mm) & $27.2(6.6)$ & $24.4(7.8)$ & $24.2(7.9)$ & $29.7(7.3)$ & 0.31 \\
\hline \multicolumn{6}{|l|}{ Type of disease ${ }^{* \dagger}$} \\
\hline Benign disease & 1 & 3 & 5 & 6 & 0.32 \\
\hline GI-cancer & 3 & 2 & 2 & 2 & \\
\hline Gyn-cancer & 3 & 4 & 4 & 6 & \\
\hline Other cancer & 1 & 2 & 0 & 0 & \\
\hline
\end{tabular}

BMI: Body mass index; ASA: American Society of Anesthesiologists physical status score. $\dagger$ Benign disease, $\mathrm{n}=15$; GI: gastrointestinal tract cancer, $\mathrm{n}=9$; Gyn: gynecological cancer, $\mathrm{n}=17$; other malignancy, $\mathrm{n}=3$.

However, no differences were detected in the hs-CRP values between the control and the three active groups preoperatively and after operation (Table III).

\section{Discussion}

Reactive oxygen species are products containing oxygen molecules that can regulate immune responses and cell signaling. In addition, they have potential to cause harm by damaging both nuclear and mitochondrial DNA, RNA, lipids, and proteins. These reactions lead to mutations and genomic instability (12). These products can also modulate signaling molecules and alter functions of enzymes and proteins involved in carcinogenesis (12). To avoid cell damage, the living cell needs defense mechanisms against free radicals such as oxygen, hydrogen peroxide and superoxide. These protective functions maintain balance between intracellular reactive species and antioxidant activity. All three known SODs are responsible for the reaction where superoxide is dimutated into $\mathrm{H}_{2}$ and $\mathrm{O}_{2}(2,3)$.

In this study, we sought answers to build a bridge between oxidative stress and inflammation in relation to postoperative pain. The primary end-point of our study was to examine postoperative pain $\left(\mathrm{NRS}_{24}\right)$ and the plasma level of SOD1 in patients undergoing midline laparotomy randomized into the control group or to different RSB analgesia groups. Each study patient had an intravenous oxycodone pump for patientcontrolled analgesia.

The main hypothesis of our study was whether the postsurgery placement of the RSB reduced oxidative stress and pain following surgery in patients with benign disease and cancer.
Table II. Postoperative alteration of plasma high-sensitivity C-reactive protein (hs-CRP), glutathione peroxidase (GPX1) and superoxide dismutase (SOD1) levels in all groups combined (all patients). Plasma marker levels were measured before operation (PRE), immediately after operation (POP1), and 24 hours after operation (POP2). Median (interquartile range) values are shown. The Wilcoxon signed-rank test was used.

\begin{tabular}{lccc}
\hline Marker & Time point & All patients & $p$-Value \\
\hline hs-CRP $(\mathrm{mg} / \mathrm{l})$ & PRE & $3.3(0.85-12.9)$ & \\
& POP1 & $4.8(0.8-46.9)$ & $0.274^{\mathrm{a}}$ \\
& POP2 & $186.9(97.2-337)$ & $<0.001^{\mathrm{b}}$ \\
GPX1 $(\mathrm{pg} / \mathrm{ml})$ & PRE & $13.2(7.7-19.7)$ & \\
& POP1 & $11.9(8.1-22.8)$ & $0.282^{\mathrm{a}}$ \\
& POP2 & $8.7(5.3-15.5)$ & $0.006^{\mathrm{b}}$ \\
SOD1 $(\mathrm{pg} / \mathrm{ml})$ & PRE & $164.0(114-212)$ & \\
& POP1 & $185.0(137-301)$ & $0.007^{\mathrm{a}}$ \\
& POP2 & $105.0(86-176)$ & $<0.001^{\mathrm{b}}$ \\
\hline
\end{tabular}

${ }^{\mathrm{a}} v s$. PRE; ${ }^{\mathrm{b} v s .}$ POP1.

We earlier reported a statistically significant inverse correlation between the individual plasma values of hs-CRP and $8-\mathrm{OHdG}$ and significant difference in GPX1 value preoperatively in patients with cancer compared to those with benign disease (9-11, 13-15). We reported a significant correlation in the satisfaction for pain treatment $\left(\mathrm{NRS}_{24}\right)$ and plasma concentrations of anti-inflammatory cytokine interleukin-10 (IL10) and pro-inflammatory cytokine IL1 $\beta$ postoperatively. In this study, we found a trend for an inverse correlation between the individual values of the $\mathrm{NRS}_{24}$ and 
Table III. Plasma high-sensitivity C-reactive protein (hs-CRP), glutathione peroxidase (GPX1) and superoxide dismutase (SOD1) levels in the four study groups. Plasma levels were determined before operation (PRE), immediately after operation (POP1) and 24 hours after operation (POP2). Median (interquartile range) values are shown. The Kruskall-Wallis test was used.

\begin{tabular}{|c|c|c|c|c|c|c|}
\hline \multirow[b]{2}{*}{ Marker } & \multirow[b]{2}{*}{ Time point } & \multicolumn{5}{|c|}{ RSB method } \\
\hline & & Control & Single & Repeated & Continuous & $p$-Value \\
\hline \multirow[t]{3}{*}{ hs-CRP (mg/l) } & PRE & $3.48(1.2-13.0)$ & $4.65(0.89-48.23)$ & $1.20(0.59-4.00)$ & $6.45(0.62-73.54)$ & 0.591 \\
\hline & POP1 & $6.6(1.0-13.2)$ & $9.18(2.9-83.3)$ & $2.30(0.59-5.13)$ & $25.9(1.03-231.8)$ & 0.289 \\
\hline & POP2 & $246.1(82.2-469.5)$ & $189.5(96.2-349.0)$ & $206.5(81.5-284.4)$ & $159.0(79.3-478.9)$ & 0.875 \\
\hline \multirow[t]{3}{*}{ GPX1 (pg/ml) } & PRE & $12.05(8.8-19.2)$ & $10.70(5.4-16.7)$ & $19.17(6.7-22.0)$ & $17.00(9.5-18.9)$ & 0.503 \\
\hline & POP1 & $11.90(8.20-36.4)$ & $9.40(6.1-23.0)$ & $15.10(6.5-29.8)$ & $15.10(10.2-20.6)$ & 0.775 \\
\hline & POP2 & $10.57(5.8-16.3)$ & $5.13(3.2-9.2)$ & $8.63(5.3-19.2)$ & $12.29(6.7-19.6)$ & 0.032 \\
\hline \multirow[t]{3}{*}{ SOD1 (pg/ml) } & PRE & $150(101-288)$ & $146(96-287)$ & $171(113-216)$ & $168(128-199)$ & 0.922 \\
\hline & POP1 & $281(144-522)$ & $160(101-305)$ & $184(123-275)$ & $243(151-292)$ & 0.392 \\
\hline & POP2 & $119(93-202)$ & $87(59-105)$ & $116(88-171)$ & $167(94-217)$ & 0.138 \\
\hline
\end{tabular}

plasma SOD1 values postoperatively in patients with benign disease and those with cancer.

The significant enhancement in the level of SOD marker but not in the GPX marker immediately after midline laparotomy may be a new finding, although the rise in the SOD level following surgery is in line with the results of Turker et al. in patients undergoing open heart surgery with cardiopulmonary bypass (16). In another study, the SOD marker levels were found to be increased 1 week postsurgery, while the GPX level stayed markedly lower than that before surgery (17).

Postoperatively, the antioxidative SOD1 and GPX1 levels in plasma were lowest in the single-dose group, lower than in the control group. This may be a true sequence of local oxidative stress caused by the local analgesic agent (18). Repeated analgesic blocks, and continuous blocks in particular, may cause a rise in the concentration of analgesic (levobupivacaine) in the plasma following surgery. This in turn has anti-inflammatory properties thus also attenuating oxidative stress, postoperatively. Although the exact mechanism of action remains unclear, it could explain the tendency towards a better anti-oxidative SOD1 level and less pain at rest 24 hours postoperatively. On the other hand, proper treatment of the incisional pain also diminishes oxidative stress.

Although the limitation of this study is the small sample size, the patients in the control group had no levobupivacaine injected and they were blinded using similar wound dressing to that for the patients in the active groups. Therefore, it is unlikely that there is a study bias from the local anesthetic used or placement of the RSB catheters, and it is possible to compare the control group with the active groups. Interestingly, recent studies have demonstrated that lidocaine and levobupivacaine may have anti-inflammatory effects when used perioperatively (19) and they may also modulate the immune system (20).
Table IV. Plasma high-sensitivity C-reactive protein (hs-CRP), glutathione peroxidase (GPX1) and superoxide dismutase (SOD1) levels in patients with benign disease $(n=15)$ and those with cancer $(n=29)$. Plasma levels were measured before operation (PRE), immediately after operation (POP1) and 24 hours after operation (POP2). Median (interquartile range) values are shown. The Mann-Whitney U-test was used.

\begin{tabular}{lcccc}
\hline Marker & $\begin{array}{l}\text { Time } \\
\text { point }\end{array}$ & Benign & Cancer & $p$-Value \\
\hline hs-CRP (mg/l) & PRE & $2.20(0.6-3.8)$ & $4.7(0.9-61.6)$ & 0.160 \\
& POP1 & $2.50(0.6-6.1)$ & $8.61(0.8-60.9)$ & 0.255 \\
& POP2 & $136.0(62.2-254.5)$ & $192.2(104.4-372.9)$ & 0.271 \\
GPX1 (pg/ml) & PRE & $18.0(12.5-22.0)$ & $10.0(5.7-17.9)$ & 0.005 \\
& POP1 & $17.3(8.7-23.1)$ & $10.7(7.5-21.3)$ & 0.253 \\
& POP2 & $11.9(6.7-24.7)$ & $8.1(5.2-12.3)$ & 0.126 \\
SOD1 (pg/ml) & PRE & $167(132-216)$ & $160(107-211)$ & 0.647 \\
& POP1 & $182(135-286)$ & $202(136-338)$ & 0.989 \\
& POP2 & $105(76-171)$ & $112(86-196)$ & 0.610 \\
\hline
\end{tabular}

In conclusion, the results suggest that midline laparotomy does significantly alter the level of the oxidative stress marker SOD1 immediately after surgery, but the levels normalizes 24 hours following surgery.

A new finding is the highly significant correlation between the individual plasma SOD1 and GPX1 values, and a trend for inverse correlation between the individual values of the $\mathrm{NRS}_{24}$ and plasma SOD1 values postoperatively in patients with benign disease and those with cancer.

\section{Conflicts of Interest}

The Authors have no conflicts of interest or financial ties to disclose. The Authors alone are responsible for the content and writing of this article. 


\section{Acknowledgements}

The study was funded by the Heikki, Aino and Aarne Korhonen foundation and the EVO-funding of the Kuopio University Hospital, Finland.

\section{References}

1 Matzuk MM, Dionne L, Guo Q, Kumar TR and Lebovitz RM: Ovarian function in Sod1 and -2 in knockout mice. Endocrinology 139: 4008-4011, 1998.

2 Estácio SG, Leal SS, Cristóvão JS, Faísca PF and Gomes CM: Calcium binding to gatekeeper residues flanking aggregationprone segments underlies non-fibrillar amyloid traits in superoxide dismutase 1 (SOD1). Biochim Biophys Acta 1854: 118-126, 2015.

3 Sea K, Sohn SH, Durazo A, Sheng Y, Shaw BF, Cao X, Taylor AB, Whitson LJ, Holloway SP, Hart PJ, Cabelli DE, Gralla EB and Valentine JS: Insights into the role of the unusual disulfide bond in copper-zinc superoxide dismutase. J Biol Chem 290: 2405-2418, 2015.

4 Yiannakopoulou ECh, Nikiteas N, Perrea D and Tsigris C: Effect of laparoscopic surgery on oxidative stress response: systematic review. Surg Laparosc Endosc Percutan Tech 23: 101-108, 2013.

5 Olakowski M, Lampe P, Mekle H and Stefanski L: Changes in activity of antioxidant enzymes in the early period after classical and laparoscopic cholecystectomy. Wiad Lek 50: 213-217, 1997 (abstract in English, article in Polish).

6 Seven R1, Seven A, Erbil Y, Mercan S and Burçak G: Lipid peroxidation and antioxidant state after laparoscopic and open cholecystectomy. Eur J Surg 165: 871-874, 1999.

7 Stipancic I, Zarkovic N, Servis D, Sabolovic S, Tatzber F and Busic Z: Oxidative stress markers after laparoscopic and open cholecystectomy. J Laparoendosc Adv Surg Tech A 15: 347-352, 2005.

8 Vodopija N, Ovcak Z, Zupancic M, Korsic L, Kramer F, Krstanoski $\mathrm{Z}$ and Parac I: Tissue ischemia due to $\mathrm{CO}_{2}$ pressure during laparoscopic radical prostatectomy. Coll Antropol 33: 7782, 2009.

9 Purdy M, Kokki M, Anttila M, Aspinen S, Juvonen P, Selander T, Kokki H, Pulkki K and Eskelinen M: Does the post-surgery placement of rectus sheath block analgesia alter the oxidative stress biomarker 8-OHdG concentrations: a randomised trial of patients with cancer and benign disease. Cancer Genomics Proteomics 13: 239-244, 2016.

10 Purdy M, Kokki M, Anttila M, Aspinen S, Juvonen P, Korhonen R, Selander T, Kokki H and Eskelinen M: Does the rectus sheath block analgesia reduce the inflammatory response biomarker IL1 ra, IL-6, IL-8, IL-10 and IL- $1 \beta$ concentrations following surgery? A randomised clinical trial of patients with cancer and benign disease. Anticancer Res 36: 3005-3011, 2016.
11 Purdy M, Kärkkäinen J, Kokki M, Anttila M, Aspinen S, Juvonen P, Kokki H, Pulkki K, Rantanen T and Eskelinen M: Does rectus sheath block analgesia alter levels of the oxidative stress biomarker glutathione peroxidase: a randomised trial of patients with cancer and benign disease. Anticancer Res 37: 897902, 2017.

12 Carini F, Mazzola M, Rappa F, Jurjus A, Geagea AG, Al Kattar S, Bou-Assi T, Jurjus R, Damiani P, Leone A and Tomasello G: Colorectal carcinogenesis: role of oxidative stress and antioxidants. Anticancer Res 37: 4759-4766, 2017.

13 Aspinen S, Kinnunen M, Harju J, Juvonen P, Selander T, Holopainen A, Kokki H, Pulkki K and Eskelinen M: Inflammatory response to surgical trauma in patients with minilaparotomy cholecystectomy versus laparoscopic cholecystectomy: a randomised multicentre study. Scand J Gastroenterol 51: 739-744, 2016.

14 Aspinen S, Harju J, Juvonen P, Selander T, Kokki H, Pulkki K and Eskelinen M: The plasma 8-OHdG levels and oxidative stress following cholecystectomy: a randomised multicentre study of patients with minilaparotomy cholecystectomy versus laparoscopic cholecystectomy. Scand J Gastroenterol 51: 1507$1511,2016$.

15 Kärkkäinen J, Aspinen S, Harju J, Juvonen P, Pulkki K and Eskelinen M: Plasma gluthathione peroxidase (GPX1) levels and oxidative stress in gallstone patients operated with two different cholecystectomy techniques: a randomized study with special reference to cancer patients. Anticancer Res 37: 6921-6928, 2017.

16 Turker FS, Dogan A, Ozan G, Kibar K and Erisir M: Change in free radical and antioxidant enzyme levels in the patients undergoing open heart surgery with cardiopulmonary bypass. Oxid Med Cell Longev 2016: 1783728, 2016.

17 Woźniak B, Woźniak A, Mila-Kierzenkowska C and Kasprzak HA: Correlation of oxidative and antioxidative processes in the blood of patients with cervical spinal cord injury. Oxid Med Cell Longev 2016: 6094631, 2016.

18 Boybeyi Ö, Gunal YD, Atasoy B, Kisa U and Aslan MK: Investigation of the effect of dorsal penile block to penile tissue. J Pediatr Urol 11: 268.e1-5, 2015.

19 Cata JP, Ramirez MF, Velasquez JF, Di A, Popat KU, Gottumukkala V, Black DM, Lewis VO and Vauthey JN: Lidocaine stimulates the function of natural killer cells in different experimental settings. Anticancer Res 37: 4727-4732, 2017.

20 Velásquez JF, Ramírez MF, Ai DI, Lewis V and Cata JP: Impaired Immune function in patients undergoing surgery for bone cancer. Anticancer Res 35: 5461-5466, 2015. 